\title{
Preliminary Notions of Arguments from Commonsense Knowledge
}

\author{
Alexandros Vassiliades \\ valexande@csd.auth.gr \\ Aristotle University of Thessaloniki \\ Institute of Computer Science, \\ Foundation for Research and \\ Technology, Hellas \\ Giorgos Flouris \\ fgeo@ics.forth.gr \\ Institute of Computer Science, \\ Foundation for Research and \\ Technology, Hellas
}

\author{
Theodore Patkos \\ patkos@ics.forth.gr \\ Institute of Computer Science, \\ Foundation for Research and \\ Technology, Hellas
}

\author{
Nick Bassiliades \\ nbassili@csd.auth.gr \\ Aristotle University of Thessaloniki
}

\author{
Antonis Bikakis \\ a.bikakis@ucl.ac.uk \\ University College London
}

\begin{abstract}
The field of Computational Argumentation is well-tailored to approach commonsense reasoning, due to its ability to model contradictory information. In this paper, we present preliminary work on how an argumentation framework can explicitly model commonsense knowledge, both at a logically structured and at an abstract level. We discuss the correlation with current research and present interesting future directions.
\end{abstract}

CCS Concepts: - Theory of computation $\rightarrow$ Automated reasoning; • Mathematics of computing $\rightarrow$ Solvers.

Keywords: Argumentation, Commonsense, Commonsense Argument, Argumentation Schemes

\section{ACM Reference Format:}

Alexandros Vassiliades, Theodore Patkos, Antonis Bikakis, Giorgos Flouris, Nick Bassiliades, and Dimitris Plexousakis. 2020. Preliminary Notions of Arguments from Commonsense Knowledge. In 11th Hellenic Conference on Artificial Intelligence (SETN 2020), September 2-4, 2020, Athens, Greece. ACM, New York, NY, USA, 4 pages. https://doi.org/10.1145/3411408.3411432

\section{Introduction}

A central and still open problem in Artificial Intelligence is the automation of the process of reasoning with commonsense knowledge. Historically, the encoding of commonsense knowledge has been primarily connected with symbolic representations, with varying degrees of progress in approaching the demands of diverse domains (see for instance [4] for a thorough analysis). The representation of such information in a logical form is not an easy task.

In this paper, we formally define initial notions towards a generic argumentation framework (AF) for agents to interact with and exchange commonsense knowledge at different levels of granularity. The framework builds on research in the field of Computational Argumentation, in order to model abstract or more structured forms of commonsense knowledge. The inherent defeasible nature of AFs, suggest argumentation as a suitable mechanism for performing automated commonsense reasoning.

The main contribution of this work is the introduction of a special type of arguments, called Commonsense Arguments (CS-Argument), which can enhance commonsense reasoning. We define how CS-arguments can be represented and what constitutes an intuitive attack, considering both the case that the argument has a certain logical representation (Section 2), as well as the case of relying on more abstract schemes (Section 3). We set preliminary work towards a comprehensive formal model for supporting or rejecting a given commonsense conclusion, through the process of argument interaction. We argue in Section 4 that distinguishing between CS-Arguments and the rest opens interesting directions for future research.

\footnotetext{
This project has received funding from the Hellenic Foundation for Research and Innovation (HFRI) and the General Secretariat for Research and Technology (GSRT), under grant agreement No 188.
} 


\section{Structuring Commonsense Arguments}

In this section, we set the foundations for modeling a framework for intelligent agents to interact with commonsense knowledge using an internal structure. For simplicity, we assume a propositional language $\mathcal{L}$, although more expressive languages can be considered; our focus is on harnessing the interaction between commonsense knowledge and exceptional cases, along with the attack relations among them, rather than on how this knowledge is represented. We use $\alpha, \beta, \gamma, \ldots$ to denote formulae and $\Delta, \Phi, \Psi, \ldots$ to denote sets of formulae. Deduction in classical propositional logic is denoted by the symbol $\vdash$ and inconsistency by the symbol $\perp$.

We assume that each agent $A$ has its own personal belief base $\Delta^{A}$ that contains formulae that are only accessible to the agent. Similarly, there is a set of public belief bases $\Delta^{C O}=$ $\left\{\Delta^{C O_{1}}, \ldots, \Delta^{C O_{n}}\right\}$ that contain information that is accessible by all agents. An agent may choose to use part of $\Delta^{C O}$, as it may not have the capacity to process the full belief bases, or it may not trust part of it, etc.

Additionally, we assume a single belief base $\Delta^{C S}$ that represents the commonly accepted by all agents commonsense knowledge about the world, i.e., the set of formulae capturing common experiences that constitute part of human intuition. A formula $\phi$ can exist in more than one of the aforementioned belief bases and the only restriction posed is that $\Delta^{A}$ and $\Delta^{C O}$ are consistent. We do not pose any restrictions on the form of information inside the knowledge bases, because we do not want these preliminary notions to tend only to a specific type of agents (i.e. those that could parse RDF triplets, free text, etc). This also holds for computing relations on the information in the knowledge bases.

A common definition of logical arguments, as given for instance in [2], separates the evidence, or support, from the claim, or conclusion. In accordance to such a definition, we next refine a logical argument to also account for commonsense knowledge:

Definition 2.1. A CS-Argument is a pair $\langle\Phi \cup \Psi, \alpha\rangle$, where $\Phi \cup \Psi$ is called the support and $\alpha$ the claim, such that: (1) $\Phi \subseteq$ $\Delta^{A} \cup \Delta^{C O},(2) \Psi \subseteq \Delta^{C S}$ and $\Psi \neq \emptyset,(3) \Phi \cup \Psi \nvdash \perp,(4) \Phi \cup \Psi \vdash \alpha$, (5) $\Phi \cup \Psi$ is minimal with respect to $\subseteq$ among the sets of formulae which satisfy items $1,2,3,4$.

For instance, a classical example of an CS-argument can be $\left\langle\left\{\phi_{1}, \phi_{2}, \phi_{1} \wedge \phi_{2} \rightarrow \alpha\right\}, \alpha\right\rangle$, with $\phi_{1}=$ "O is an apple", $\phi_{2}=$ "apples are typically red", $\phi_{1} \in \Delta^{A}, \phi_{2} \in \Delta^{C S}$ and $\alpha=$ "O is expected to be red".

As is well known, there are exceptional cases in such commonsense inferences. Different logics and numerous semantics have been proposed to model exceptions from the default behavior. Yet, a common idea shared by most models is not to question the validity of the default, rather to weaken its scope, in order to accommodate exceptional cases. Therefore, in order to facilitate a generic treatment of commonsense attacks for arguments written in a deductive form, instead of questioning the inference rules in the premises of the argument, we define a new relation that explicitly prioritises exceptional cases over commonsense statements. This enables the model to indirectly weaken the inference rule, without affecting logical inference or becoming dependent on how defaults are implemented.

Definition 2.2. A commonsense exception relation $>$ is an irreflexive and anti-symmetric binary relation that defines $\omega \in\left(\Delta^{A} \cup \Delta^{C O} \cup \Delta^{C S}\right)$ as an exception of a formula $\psi \in \Delta^{C S}$ :

$$
>=\left\{(\omega, \psi) \in\left(\Delta^{A} \cup \Delta^{C O} \cup \Delta^{C S}, \Delta^{C S}\right)\right\}
$$

For example, consider $\phi_{4}, \phi_{5}$ obtained from a public agriculture repository, where $\phi_{4}=$ "Granny Smith is an apple cultivar" and $\phi_{5}=$ "Granny Smiths are green". An agent can then define $\left(\phi_{5}, \phi_{2}\right) \in>$ (or with an abuse of notation, $\left.\phi_{5}>\phi_{2}\right)$. Such a definition does not intend to invalidate the logical validity of a commonsense formulae ( $\phi_{2}$ in this case), rather to refine its scope; as a result, the literal can still be used in formulae within the same or other arguments, as long as the exceptional conditions are not met. Relying on the exception relation, we can define a special type of attacks on commonsense arguments, in addition to the ones defined for ordinary deductive arguments.

Definition 2.3. A commonsense defeater of a commonsense argument $\langle\Phi \cup \Psi, \alpha\rangle$ is an argument $\langle\Omega, \beta\rangle$, such that: (1) $\omega>\psi$, where $\omega \in(\Omega \cup \beta)(2) \beta \vdash \neg \alpha$.

Notice that any argument can still attack the non commonsense premises and claim of a CS-Argument. As such, this modeling offers the substrate on top of which to develop a comprehensive model of argumentation for reasoning about commonsense, by identifying rational patterns of attacks that can be made on commonsense statements.

\section{Abstract Schemes for Commonsense Arguments}

In the previous section, we discussed CS-Arguments that can be structured in a deductive form. Yet, the concepts and domains that commonsense knowledge can cover are so rich that are difficult to represent symbolically, even for cases where there is in-depth understanding, e.g., space, physics and folk psychology [4]. In this section, we discuss more abstract models of argumentation for reasoning about commonsense. After all, as also argued in [8], the use of abstract frameworks is interesting, even when an underlying belief base does exist.

\subsection{A Dung's AF for CS-Arguments}

The more general case is to rely on a typical Dung-style AF (see [5]), extending it with a special type of attacks:

Definition 3.1. Given $D$ and $C S$ denoting respectively the set of all arguments and CS-Arguments $(C S \subseteq D)$, a commonsense $\mathrm{AF}$ is a pair $C S A F=(A, R)$, with (1) $A=D \cup C S$; 
(2) $R=R_{D} \cup R_{C S}$; (3) $R_{D} \subseteq D \times(D \backslash C S)$ the set of attacks to deductive, non CS-Arguments; (4) $R_{C S} \subseteq D \times C S$ the set of attacks to CS-Arguments.

Computing the extensions of a CSAF can be identical to the process for classical Dung's AFs. Still, differentiating the kinds of attacks can be useful for enabling intuitive revisions or for defining semantics particularly suited for commonsense attacks.

\subsection{Toulmin's Argumentation Model}

A more fine-grained model is Toulmin's Argument Model [12]. According to Toulmin, there should be data (grounds) that support a given claim and there should be at least one warrant to support the relation among the data and the claim. The warrant is closely related to commonsense knowledge, which can further be backed up with additional support, justification or reasons through the backing component of Toulmin's model.

Consider an example, borrowed from [4], expressing plausible inference from frequencies: Pierre lives in Paris (data) and since most residents of Paris can speak French (warrant), it is expected that Pierre can speak French (claim). The backing can include reasons, such as that in most cities the majority of the residents speak the official language of the country they live in, and the official language of the French Republic is French, according to art. 2 of the French Constitution, etc. According to Toulmin, the strength of an argument is captured by the qualifier, a component that can be a number or a symbolic value. This is strongly related to the rebuttal. In the case of CS-Arguments, the rebuttal can list the known exceptions of the commonsense truth expressed in the warrant.

Proposition 3.2. Toulmin's Argumentation Model can be mapped into the CSAF, and vice versa.

Method: We can map any argument from Toulmin's Argumentation Model into a CS-Argument $\langle\Phi \cup \Psi, \alpha\rangle$, if we let $\Phi$ be the data and backing part of the argument, $\Psi$ the warrant, and commonsense exceptions the rebuttal. In the previous example, we get $\Phi=\left\{\phi_{1}, \phi_{2}, \phi_{3}\right\}$ where $\phi_{1}=$ "Pierre lives in Paris" (data), $\phi_{2}=$ "In cities the majority of residents speak the countries official language" and $\phi_{3}=$ "The official language of French Republic is French" (backing). $\Psi=\left\{\psi_{1}\right\}$, where $\psi_{1}=$ "Residents of Paris can speak French" (warrant). Using this transformation we can construct the CS-Argument $\langle\Phi \cup \Psi, \alpha\rangle$, where $a=$ "Pierre is expected to speak French". A commonsense defeater is an argument $\langle\Omega, \beta\rangle$ that contains the formulae (rebuttal) $\phi_{4}=$ "Pierre moved from the US to Paris last month.", $\phi_{4} \gg \psi_{1}$.

\subsection{Argument Schemes}

Patterns for constructing arguments, as suggested by Walton et al. [13] also offer the possibility to represent more abstractly CS-Arguments, yet in a way that allows for refining proper attack relations. Argument schemes are accompanied by a set of critical questions to restrict the ways a particular scheme may fail. There are different schemes that can be adapted for the purpose of constructing arguments related to commonsense knowledge, in a way similar to schemes adapted for arguments related to trust (see e.g., [11]).

Argument from Commonsense Classification: All F's are typically classified as G's. $\alpha$ is an F, therefore $\alpha$ is a G. As an example, consider that all birds are typically classified as animals that can fly; since Tweety is a bird, it is expected to be an animal that can fly. From the commonsense perspective, we analyze if $\alpha$ falls into an exceptional category.

Proposition 3.3. Argument from Commonsense Classification can be mapped into the CSAF, and vice versa.

Method: In this case, let $\langle\Phi \cup \Psi, \alpha\rangle$ be an CS-Argument. If we consider as commonsense knowledge $\Psi$ the part that states: All F's are typically classified as G's, and $\Phi$ the personal and public knowledge that the entity $\alpha$ is an F. We can map the scheme into the CSAF. In the example, $\phi_{1}=$ "Tweety is a bird" $\in \Phi$, and $\psi_{1}=$ "Birds are animals that can fly" $\in \Psi$, the CS-Argument $\langle\Phi \cup \Psi, \alpha\rangle$ is constructed where $\alpha=$ "Tweety is expected to fly". An argument $\langle\Omega, \beta\rangle$ with the formulae $\phi_{2}=$ "Tweety is a chicken" in its support is a commonsense defeater for $\langle\Phi \cup \Psi, \alpha\rangle$, because $\phi_{2} \gg \psi_{1}$.

Argument from Established Commonsense Rule: If carrying out $\mathrm{A}$ is the established rule for $x$, then $x$ must carry out A. Let, Tweety be a bird and birds typically fly. Therefore, Tweety is expected to fly. Of course, there are exceptions to the commonsense rule "birds typically fly", such as Tweety is a chicken. The established rule tries to define a universal relation, but in some cases there are exceptions that cannot be tackled.

Proposition 3.4. Argument from Established Commonsense Rule can be mapped into the CSAF, and vice versa.

Method: For this case, given a CS-Argument $\langle\Phi \cup \Psi, \alpha\rangle$. We consider as personal (or public) knowledge $\Phi$ the fact that an entity $x$ is instance of a category that the established rule $A$ applies, and as commonsense knowledge $\Psi$ the part which states that $x$ must carry the rule $A$. In the example, $\Phi=\left\{\phi_{1}\right\}$ where $\phi_{1}=$ "Tweety is a bird", and $\Psi=\left\{\psi_{1}\right\}$ where $\psi_{1}=$ "Birds typically fly", therefore the CS-Argument $\langle\Phi \cup \Psi, \alpha\rangle$ supports $\alpha=$ "Tweety can fly". A commonsense defeater, in this case is the argument $\langle\Omega, \beta\rangle$ that has the formulae $\phi_{2}=$ "Tweety is a chicken" in its support, $\phi_{2} \gg \psi_{1}$.

Argument from Popularity: If everyone in a particular reference group accepts A, then A is presumably true. For example, Tweety is a bird and birds typically live in trees. Therefore, Tweety presumably lives in a tree. Notice that it is not clear even that statistically most birds live in tree; e.g., chickens, that are numerous, do not.

Proposition 3.5. Argument from Popularity can be mapped into the CSAF, and vice versa. 
Method: Given a CS-Argument $\langle\Phi \cup \Psi, \alpha\rangle$. We consider $\Phi$ as the knowledge that A holds for an entity, and $\Psi$ as the knowledge that a particular reference group accepts $A$, and A is presumably true.

Argument from Popular Practice: If a large majority does or acts though $A$, then $A$ is the correct thing to do (unless is an exception). For instance, many people do jogging because it provides wellness to their bodies, and Pierre does jogging, Therefore, Pierre is expected to have body wellness. An exception to this common practice is that Pierre has heart arrhythmia.

Proposition 3.6. Argument from Popular Practice can be mapped into the CSAF, and vice versa.

Method: Here given a CS-Argument $\langle\Phi \cup \Psi, \alpha\rangle$. We take $\Phi$ the knowledge that an entity performs or acts though a popular practice $A$, and $\Psi$ the knowledge that $A$ is the correct thing to do.

\section{Discussion and Conclusion}

The formalisation of default assumptions and of relevant exceptions using some form of argumentation logic can been seen as a natural fit for argumentation systems, due to the ability of the latter to reason with contradictory information. Our proposed CSAF builds on this correlation, making explicit the commonsense notions at the conceptual level. This way, it becomes possible to both to take advantage of the progress in sub-fields of Computational Argumentation, as well as to identify new areas that require research, particularly suitable for the needs of commonsense reasoning. Other studies approach commonsense reasoning with AF using preference rules [3], Event Calculus [1], and Web resources [7], without explicitly distinguishing commonsense from other types of knowledge.

The research about enthymemes for instance, i.e., partially specified, logically invalid arguments, is highly relevant. As pointed out in [6], entymemes can capture more accurately real human arguments, as they typically rely on statements not explicitly spelled out, due to the assumption that they belong to some common knowledge. In this sense, commonsense knowledge constitutes probably the most common type of statements missing in an enthymeme. Similarly, as a human is more likely to be persuaded by an argument that is in line with her own commonsense knowledge, our framework is also related to research on persuasive argumentation and on dialectical systems.

At the same time, new directions explicitly tailored to commonsense reasoning can be envisioned. By distinguishing between CS-Arguments and the rest, our next reasonable step is to model intuitive extensions of argumentation graphs that take into consideration the different types of attacks that can be applied. Already, recognising and dealing with mistaken attacks is attracting the attention within enthymeme-based
AFs (e.g., [8]). Note that in a typical logical AF, attacks between arguments are not questioned, as they stem from the logical inference relation. On the contrary, our exception relation does not define a logical correlation, but rather a prioritisation, among formulae.

Weighted counterparts of AF [9], as well as hierarchical related AF [10], assign strength values to arguments, in order to decide which argument is stronger. Such approaches can provide useful properties in a CS-based framework, yet our modeling is meant to cover more generic cases, where the existence of exceptions do not work quantitatively for the argument, rather they show some sub case where the argument does not hold. We define CS-Arguments in this manner because we want them to be the most commonly used and known argument in a conversation. Elaborating with CS-Arguments and weights, based on the context of the conversation is left as future work.

To conclude, we presented preliminary work towards an AF for reasoning with commonsense knowledge. We defined the notions of CS-Arguments and exceptions, both at a structured and at a more abstract level, opening interesting directions for future extensions.

\section{References}

[1] Sofia Almpani and Petros S Stefaneas. 2017. On Proving and argumentation.. In AIC. 72-84.

[2] Philippe Besnard and Anthony Hunter. 2001. A logic-based theory of deductive arguments. Artificial Intelligence 128, 1-2 (2001), 203-235.

[3] Kristijonas Čyras. 2016. Argumentation-based reasoning with preferences. Springer.

[4] Ernest Davis. 2017. Logical Formalizations of Commonsense Reasoning: A Survey. Journal of Artificial Intelligence Research 59 (2017), 651-723.

[5] Phan Minh Dung. 1995. An argumentation-theoretic foundation for logic programming. The fournal of logic programming 22, 2 (1995), 151-177.

[6] Anthony Hunter. 2007. Real Arguments Are Approximate Arguments. In Proceedings of the 22nd National Conference on Artificial Intelligence - Volume 1 (AAAI'07). AAAI Press, Palo Alto, California, 66-71.

[7] Jonathan Kobbe, Juri Opitz, Maria Becker, Ioana Hulpus, Heiner Stuckenschmidt, and Anette Frank. 2019. Exploiting background knowledge for argumentative relation classification.

[8] Jean-Guy Mailly. 2016. Using Enthymemes to Fill the Gap between Logical Argumentation and Revision of Abstract Argumentation Frameworks. CoRR abs/1603.08789 (2016), 95-106.

[9] Diego C Martınez, Alejandro Javier Garcıa, and Guillermo R Simari. 2008. An abstract argumentation framework with varied-strength attacks. In Proceedings of 11th Knowledge Representation and Reasoning Conference (KR'08). 135-144.

[10] Sanjay Modgil. 2006. Value based argumentation in hierarchical argumentation frameworks. COMMA 144 (2006), 297-308.

[11] Simon Parsons, Katie Atkinson, Zimi Li, Peter McBurney, Elizabeth Sklar, Munindar Singh, Karen Haigh, Karl Levitt, and Jeff Rowe. 2014. Argument schemes for reasoning about trust. Argument \& Computation 5, 2-3 (2014), 160-190.

[12] Stephen E. Toulmin. 2003. The Uses of Argument (2 ed.). Cambridge University Press, Cambridge, UK.

[13] Douglas Walton, Christopher Reed, and Fabrizio Macagno. 2008. Argumentation schemes. Cambridge University Press, UK, Cambridge. 